\title{
Research on Compressed Sensing Data Acquisition Method in Cold Chain Logistics
}

\author{
Rui Luo ${ }^{1, a}$, Zihong Zhang ${ }^{2, b^{*}}$ and Wei Xiong ${ }^{3, c}$ \\ ${ }^{123} \mathrm{HongHe}$ University, MengZi, YunNan,China \\ a35660520@qq.com, b56090733@qq.com, c459353359@qq.com
}

Keywords: Bi-orthogonal avelet transforms basis; Cold chain logistics; Data acquisition; OMP

\begin{abstract}
The article designs and implements the hardware and software of cold chain logistics quality perception based on Internet of things, and establish a sparse compression sampling model based on biorthogonal wavelet transform base, wireless real-time sparse compression and transmission model and the reconstruction model based on orthogonal matching pursuit (OMP), and then form a compressed sensing data acquisition method for constructing the cold chain logistics quality perception data. Finally, the authors verified the data volume in the data transmission by using the cold chain logistics supply chain of fresh grapes, which can effectively reduce the wireless communication load,and realize the efficient collection and transmission of food cold chain logistics information. The algorithm can improve the reliability and accuracy of information, and form the compressed sensing data set of cold chain logistics quality perception.
\end{abstract}

\section{Introduction}

In the process of cold chain logistics, we should not only maintain the low temperature environment needed for the product, but also need integrated preservation, storage of goods and real-time monitoring and management, so as to ensure that cold chain logistics is in real time monitoring and traceabilityin the whole process. The cold chain logistics environment parameters are complex and changeable, and the temperature, relative humidity, gas and other parameters are interlaced together to show strong multi source coupling characteristics in the cold chain logistics environment.Based on Wireless Sensor Networks, the cold chain logistics data acquisition method can achieve real-time monitoring of cold chain logistics and improve the safety and transparency in cold chain logistics process. But the wireless sensor network contains a large number of sensor nodes, each sensor node will collect a large amount of perceptual data, however, the processing capability, storage space, communication bandwidth and energy of the sensor nodes are very limited, and it cannot manage and store a large amount of data like the traditional information system. At the same time, the transmission of large amounts of data makes the nodes consume too many resources. Therefore, it is necessary to find efficient data acquisition and transmission technology to adapt to its limited resources.

\section{The Basic Principle of Compressed Sensing}

Compressed Sensing (CS) was first proposed in 2006 by CandSs, which aims to reduce the sampling frequency, thus saving data storage space, transmission and computing, and so on, Using the combined high-speed sampling and compression transformation process, the algorithm first performs sparse and low speed sampling on the compressed signal, and then reconstructs the sparse compression signal, which can effectively reduce the amount of data in the real-time acquisition process of WSN data and improve the efficiency of WSN data acquisition and transmission. The process is shown in fig. 1. 


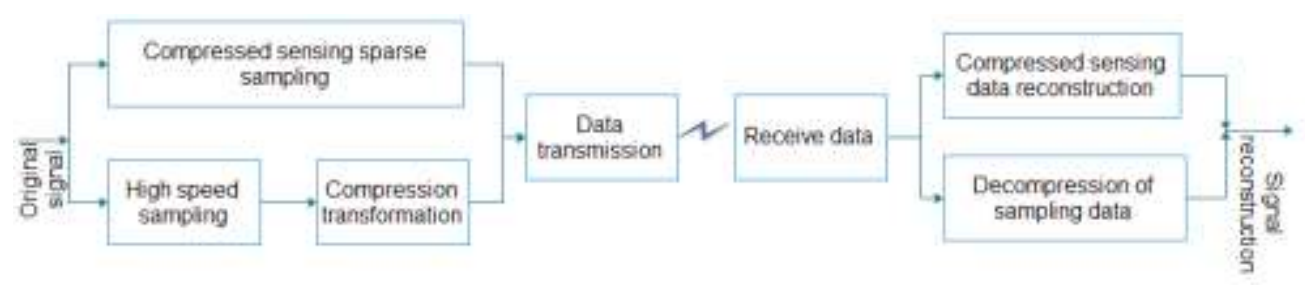

Figure. 1 The principle of perceptual data acquisition process

Sparse compression sampling for data. $N$ dimensional perceptual data vector $X=(X(1)$, $\mathrm{X}(2), \ldots, \mathrm{X}(\mathrm{N})) \mathrm{T} \in \mathrm{RN}$, its sparse representation is shown in Eq. 1.

$$
\hat{X}=\arg \min \|X\|_{0} \text { s.t. } \Phi X=Y
$$

In the formula, $\Psi=\left[\Psi_{1}, \Psi_{2} \ldots \Psi_{N}\right], \Psi \in R^{N_{X} N_{Y}}$ is $\mathrm{N} * \mathrm{~N}$ standard orthonormal sparse matrix, $\Psi_{i}$ is the directed quantum set of the sparse matrix of the standard orthogonal basis; $\mathrm{S}$ is $\mathrm{N}$ dimension sparse representation coefficient set of perceived data vector X. $S_{i}$ is vector elements, $S=\left[s_{1}, S_{2} \ldots s_{i}\right]^{T}$ and $S \in R^{N}$

Using random normal matrix to observe the perceptual data, we can get the sparse compression sampling of the perceptual data, as shown in Eq. 2.

$Y=\Phi X$

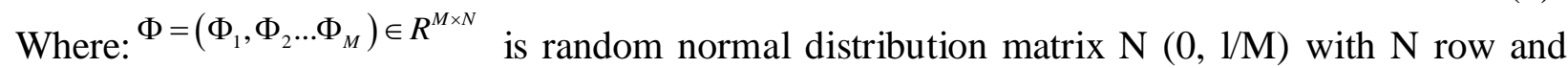
$\mathrm{M}$ row; $\mathrm{Y}$ is $\mathrm{M}$ dimension sparse compressed sampling sensing data set.

Reconfiguration of received data. Using the 0 norm sense optimization problem, we can solve the reconstruction of perceptual data after sparse compressed sampling, as shown in Eq. 3.

$$
\hat{X}=\arg \min \|X\|_{0} \text { s.t. } \Phi X=Y
$$

Wherein: $\hat{X}$ is the exact or approximate value of the original perceptual data $\mathrm{X}$.

Performance evaluation model. (1) Normalized mean square error value(NMSE)

For the reconstruction error after sparse compression sampling, we can use the normalized mean square error value (NMSE) to determine the error between the reconstructed perceptive data and the original perceptual data, as shown in Eq. 4.

$$
N M S E=\frac{\left\|\hat{x}_{j}(n)-x_{j}(n)\right\|_{p}}{\left\|x_{j}(n)\right\|_{p}}
$$

Where: $x_{j}(n) \hat{x}_{j}(n)$ is the $\mathrm{j}$ perception value corresponding to original perception data and sparse compressed sampling sensing data reconstruction.

(2) Data compression rate

The compression rate is used to measure the compression efficiency between the reconstructed perceptive data and the original perceptual data. The perceptual data compression rate is expressed as a symbol $\rho$, as shown in Eq. 5 .

$$
\rho=\frac{N-M}{N} \times 100 \%
$$

Among them, $\mathrm{N}$ is the total number of raw perception data, and $\mathrm{M}$ is the observed number of perceived data. 


\section{Quality aware sparse compressed sampling and reconfiguration model for cold chain logistics}

The principle of sparse compression sampling. Data collection process of cold chain logistics compression perception is as shown in fig. 2. According to the previous hardware and software design of Internet of things, the cold chain logistics quality perception process is: The wireless sensor node of the Internet of things completes the synchronization of the whole WSN network according to the network synchronization instructions received by the convergence node of the Internet of things; Secondly, the cold chain logistics perception data is collected and transmitted to the sink node in real time according to the data acquisition instructions. Third, the interconnection node of the Internet of things is responsible for collecting real-time sensing data $\mathrm{X}$ transmitted by wireless sensor nodes and carrying out sparse compression sampling, and transmitting the cold chain logistics perception data $\mathrm{Y}$ after sparse compression sampling to the Internet of things monitoring service center through GPRS. Fourth, when the monitoring service center receives the sparse compressed sampling perception data, the reconstruction of the perceptual data is completed according to the corresponding data reconstruction model, and the subsequent data processing and analysis are carried out.

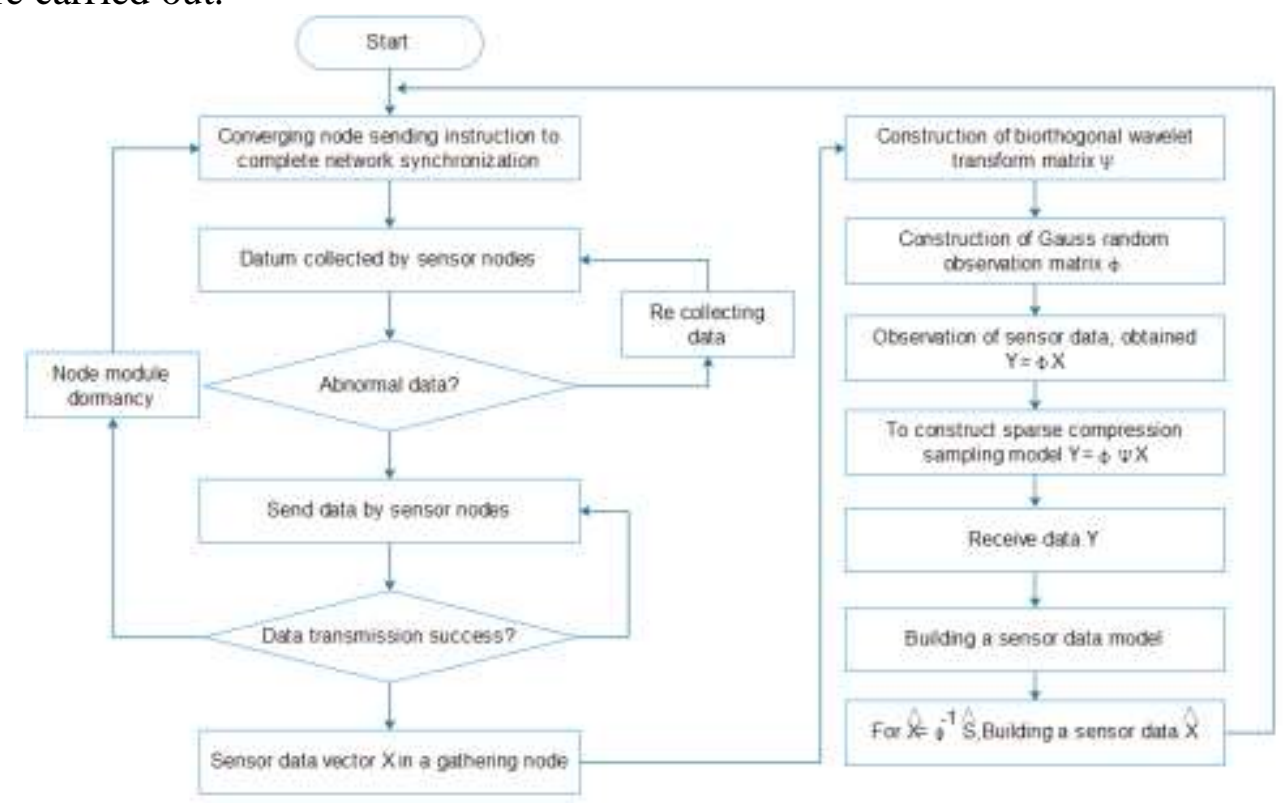

Figure 2. Data collection process of cold chain logistics compression perception

The process of quality perception data reconstruction in cold chain logistics. According to the contrastive analysis of the reconstruction method for compressed sensing data, and combining the cold chain practice, we adopt orthogonal matching pursuit (OMP) method;it can effectively and quickly solve the cold chain sparse compression sensing data reconstruction. The basic idea is as shown in Fig. 3. 


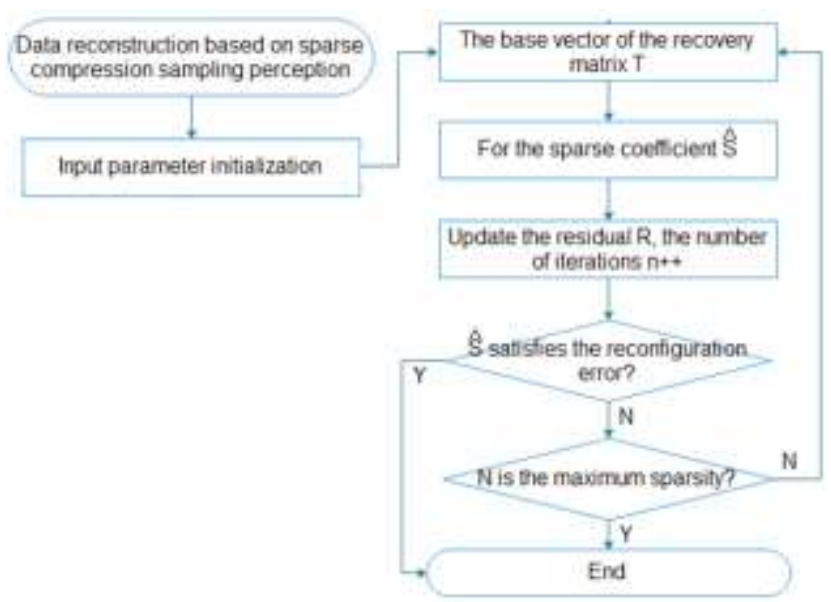

Figure 3. Flow chart of data refactoring model

\section{Verification of Compressed Sensing Performance}

During the compression sensing data acquisition process for cold chain logistics, the total number of sensing data for temperature and relative humidity is 2160 in the sensor node in $1 \mathrm{~min}$, and the observation data $\mathrm{M}$ is 512 in the sparse compression sampling processing. The normalized mean square error value (NMES), the mean absolute error value (MAE) and the average relative error value (MRE) defined by formula 4 are used to measure the reconstruction error of the perceptual data. The data compression rate defined by formula 5 is used to measure data compression efficiency. We studied the constant temperature and temperature change of fresh grapes in cold chain logistics, itscompressed sensing performance between the reconstructed environmental perception data and the original collected data is shown in Table 1.

Table 1 Compression perception performance table in cold chain logistics

\begin{tabular}{|c|c|c|c|c|c|c|}
\hline $\begin{array}{l}\text { Environmental } \\
\text { conditions }\end{array}$ & $\begin{array}{l}\text { Cold chain } \\
\text { object }\end{array}$ & $\begin{array}{l}\text { Perceptual } \\
\text { parameter }\end{array}$ & $\begin{array}{l}\text { NMES } \\
(\%)\end{array}$ & MAE & $\begin{array}{l}\text { MRE } \\
(\%)\end{array}$ & $\begin{array}{c}\text { Data compression } \\
\text { rate }(\%)\end{array}$ \\
\hline \multirow[b]{2}{*}{$\begin{array}{c}\text { Constant temperature } \\
\text { condition }\end{array}$} & \multirow[b]{2}{*}{$\begin{array}{l}\text { Fresh } \\
\text { grape }\end{array}$} & Temperature & 3.97 & $0.02^{2} \mathrm{C}$ & 9.52 & 76.3 \\
\hline & & $\begin{array}{l}\text { Relative } \\
\text { humidity }\end{array}$ & 0.048 & $0.18 \%$ & 0.21 & 76.3 \\
\hline \multirow[b]{2}{*}{$\begin{array}{l}\text { Variable temperature } \\
\text { condition }\end{array}$} & \multirow[b]{2}{*}{$\begin{array}{l}\text { Fresh } \\
\text { grape }\end{array}$} & Temperature & 4.09 & $0.72^{\circ} \mathrm{C}$ & 17.61 & 76.3 \\
\hline & & $\begin{array}{l}\text { Relative } \\
\text { humidity }\end{array}$ & 0.10 & $0.21 \%$ & 0.31 & 76.3 \\
\hline
\end{tabular}

*. Mean absolute error (MAE) and mean relative error (MRE).

\section{Conclusions}

The results of the cold chain logistics compression perception reconstruction performance analysis show that the cold chain logistics compressed sensing data acquisition method can accurately and effectively realize the reconstruction of cold chain environment sensing data after sparse compression sampling. It also reflects the changes of the cold chain environmental perception data in real time, which ensures the quality of the cold chain logistics process and improves the transparency, security and traceability of the cold chain logistics. The compression rate of compressed sensing data is $76.30 \%$. The reconfiguration errors of temperature and relative humidity for fresh grapes are respectively $3.97 \%$ and $0.084 \%$ in cold chain constant temperature, $4.09 \%$ and $0.10 \%$ under the variable temperature conditions.

\section{Acknowledgements}

The research was supported by scientific research fund of the Yunnan Provincial Education Department. No.2018JS476 


\section{References}

[1] Brunelli D and Caione C: Sensors, Vol. 15 (2015) No.7, p.16654.

[2] Larios D F, BarbanchoJ, RodriguezG,et al: IET communications, Vol. 6 (2012) No.14, p.2189.

[3] Pinto A R, Montez C, Araujo G, et al: InformationFusion, (2014) No.15, p.90.

[4] Barcelo-Llado J E, Perez A M and Seco-Granados G: IEEE Sensors Journal, Vol. 12 (2012) No.9, p.2799. 